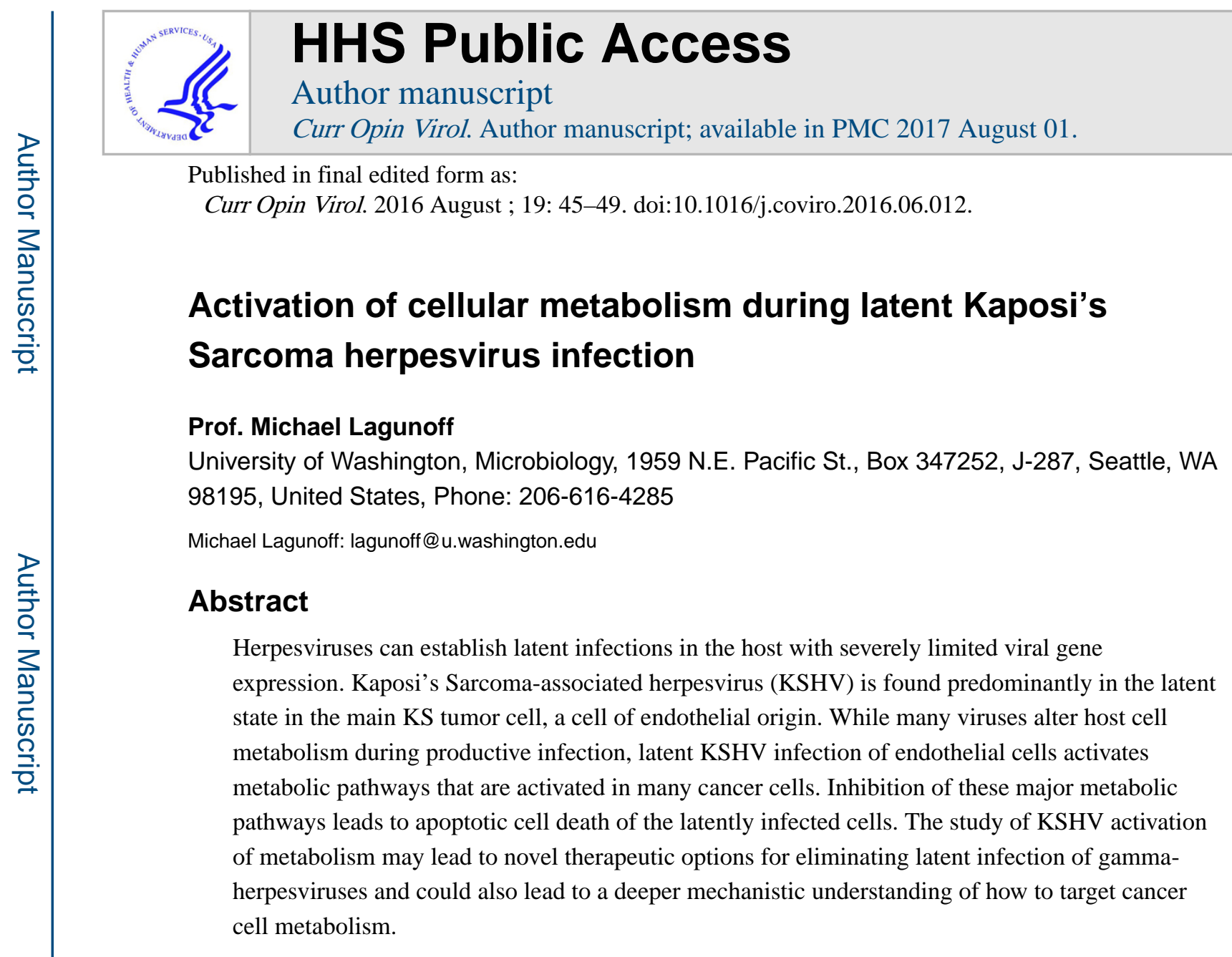

\title{
Introduction
}

A common feature of all herpesviruses is the ability to establish life long latency in their host. During latent infection, herpesvirus genomes are almost always maintained as episomes where only a very limited number of viral genes are expressed. Latent herpesviruses do not replicate via a rolling circle mechanism, as occurs during lytic replication, and new viral particles are not produced. Therefore, it is assumed that latency is a fairly quiescent infection with little damage to the host cell. Herpesviruses have evolved to encode functions to keep the latently infected host cell alive [1,2]. While Human alphaherpesviruses establish latency in non-dividing cells, the beta and gamma herpesviruses establish latency in proliferating cells and therefore, they must encode proteins to maintain the viral genome during cell division [3-5]. Thus the viral genes expressed during latency allow for long-term latency and contributes to the ability of the herpesviruses to maintain infection for the life of the host.

The two human gamma-herpesviruses, EBV and KSHV are both associated with B-cell lymphomas and tumors of other cell types during latent infection. Latent EBV infection is associated with the majority of Burkitt's lymphoma (BL) in Africa and some BL in

Publisher's Disclaimer: This is a PDF file of an unedited manuscript that has been accepted for publication. As a service to our customers we are providing this early version of the manuscript. The manuscript will undergo copyediting, typesetting, and review of the resulting proof before it is published in its final citable form. Please note that during the production process errors may be discovered which could affect the content, and all legal disclaimers that apply to the journal pertain. 
developed countries. In addition to other B-cell lymphomas, EBV is also associated with nasopharyngeal carcinoma, an epithelial cell cancer common to Southeast Asia. KSHV is associated with primary effusion lymphomas and, as the name suggests, is the etiologic agent of Kaposi's Sarcoma (KS), an endothelial based tumor. KS is the most common tumor of AIDS patients worldwide and is among the most common tumors overall in parts of subsaharan Africa [6]. As both latent KSHV and EBV infection can lead to tumor formation they likely encode oncogenic functions during this phase.

\section{Cancer Cell Metabolism}

In the 1920s, Otto Warburg first proposed that metabolic changes play a major role in cancer [7]. He found that cancer cells have increased levels of glucose uptake and glycolysis leading to increased production of lactic acid. Increased glycolysis is commonly accompanied by a decrease in oxygen consumption due to decreased oxidative phosphorylation in the mitochondria. These changes in metabolism, dubbed the Warburg effect, generally occur in most, if not all, cancer cells [8]. Fatty acid synthesis is also commonly increased in cancer cells, where there is a switch to, or increase in, de novo fatty acid synthesis generated from citrate [8,9]. In cancer cells, tri-carboxylic acid (TCA) cycle intermediates are depleted by the utilization of glucose for lactic acid production and citrate for fatty acid synthesis. Anapleurosis is a process by which TCA cycle intermediates can be supplemented from other sources, namely glutamine. Glutamine is the second most abundant carbon source in the blood and cancer cells are often glutamine addicted. Many cancer cells increase glutamine uptake and require glutaminolysis, the breakdown of glutamine to provide increased levels of the TCA cycle intermediate alpha-ketoglutarate $[8,10]$. It has been recently appreciated that changes in cellular metabolism may be a driver in oncogenesis not simply a cellular adaptation $[11,12]$. While these three pathways, shown in figure 1, are clearly important for cancer cell growth, the exact requirements for these metabolic switches are not clear. A better understanding of how cells switch to cancer cell metabolism could be helpful in creating novel cancer therapies through the application of metabolic inhibitors $[13,14]$. Studies of how viruses alter cell metabolism may lead to novel findings about cancer cell metabolism. In particular, the study of latent infection, where the cell is kept alive long-term, will be relevant to the study of altered metabolism during oncogenesis.

\section{KSHV induces the Warburg effect during latency}

KSHV latent infection of endothelial cells in culture induces the Warburg effect. Latent KSHV infection significantly increases glucose uptake of endothelial cells and induces the production of lactic acid, thereby rapidly acidifying the media of latently infected cells [15]. There is an increase in the expression of Hexokinase 2, the first rate-limiting step of glycolysis, as well as the expression of glucose transporter 3 [15]. Oxygen consumption of latently infected endothelial cells decreases compared to mock infected cells, indicating a decrease in oxidative phosphorylation in the mitochondria, as often accompanies the Warburg effect [15]. Importantly, inhibition of glycolysis with drugs that inhibit steps of glycolysis, including Oxamate and 2-Deoxyglucose, induce apoptosis in the latently infected cells at much higher rates than in their mock counterparts [15]. These data indicate that 
endothelial cells latently infected with KSHV induce and become dependent on the induction of glycolysis. KSHV induction of glycolysis does not occur in Human Foreskin Fibroblast cells, demonstrating there is some cell type specificity to the induction of glycolysis by KSHV [15]. KSHV induces hypoxia induced factors, HIF-1 and HIF-2 transcriptional activity in endothelial cells [16]. Inhibition of HIF blocked the induction of glycolysis by KSHV indicating that HIFs are responsible for at least some of the KSHV induction of glycolysis [17]. Primary effusion lymphoma cell lines, a KSHV induced B-cell lymphoma, EBV associated Burkitt's lymphoma cell lines and nasopharyngeal carcinoma cells all have activated glycolysis [18-20]. While clouded by the fact that these cells are derived from human cancers, they certainly raise the possibility that KSHV and EBV latency in B-cells and EBV in epithelial cells also activate glycolysis similarly to KSHV latent infection of endothelial cells. There is also evidence that EBV infection of B-cells induces glycolysis in the cells that form the EBV immortalized lymphoblastoid cells [21,22].

Latent genes from both KSHV and EBV are sufficient to induce glycolysis. KSHV expresses numerous microRNAs from 12 loci. Overexpression of a region containing 10 of these miRNA loci was sufficient to induce glycolysis in endothelial cells [23]. The latent EBV protein, LMP-1, that is expressed in specific types of EBV latency, is also sufficient to induce glycolysis $[18,24]$ though LMP-1 is not expressed in all types of EBV latency. Therefore, both KSHV and EBV have evolved to encode functions that are expressed during latency and are sufficient to activate glycolysis. Further experiments are necessary to demonstrate that these genes are necessary for viral induction of glycolysis during latency in endothelial or B-cells.

\section{KSHV activates fatty acid synthesis during latency}

A global screen of metabolite levels revealed that latent KSHV infection induces several additional metabolic changes, including products of fatty acid synthesis [25]. A massspectrometry based metabolomics screen measured the levels of nearly 200 metabolites at 48 and 96 hours post infection when latency is established. At 96 hours post-infection, nearly one-third of all the measured metabolite levels were altered, with the majority of the changes resulting in increased levels of metabolites [25]. Interestingly, greater than half of the long chain fatty acids measured in the screen were increased during latent infection. There is also an increase in lipid droplets, small organelles used for signaling and storage of fatty acids [25]. Inhibition of Fatty acid synthesis by the competitive inhibitor, TOFA, or the irreversible inhibitor, C75, led to greatly increased death of latently infected endothelial cells but not mock infected cells [25]. Infected cell death due to TOFA treatment was prevented, at least in part, through the addition of palmitic acid, the substrate for fatty acid elongation immediately downstream of where the inhibitors block. Therefore, KSHV induces and requires fatty acid synthesis for survival of the latently infected endothelial cells. In addition to high glycolytic flux, Primary effusion lymphoma cell lines also have high levels of fatty acid synthesis as compared to primary B-cells and are highly sensitive to fatty acid synthesis inhibitors indicating that fatty acid synthesis is also necessary for the survival of latently infected B-cells as well [20]. 


\section{KSHV infected cells are glutamine addicted}

Endothelial cells latently infected with KSHV induce and require glutaminolysis. Glycolysis shunts carbon to lactic acid and away from the TCA cycle and fatty acid synthesis shunts citrate from the TCA cycle to long chain fatty acid production. Therefore, by inducing both glycolysis and fatty acid synthesis, the latently infected cells become deficient in TCA cycle intermediates. The metabolomics screen indicated that there was increased glutamine in latently infected endothelial cells [25]. Through glutaminolysis, glutamine can be converted to alpha-ketoglutarate, a TCA cycle intermediate (fig. 1). Latently infected endothelial cells exhibit increased glutamine uptake and are much more sensitive to the absence of glutamine in the extracellular medium as compared to mock-infected cells [26]. The sensitivity to the absence of glutamine could be recovered by the addition of TCA cycle intermediates. Additionally, the addition of inhibitors of glutaminolysis also led to infected cell death, indicating glutamine uptake was required for filling the TCA cycle through anaplerosis [26]. The Myc family member, MondoA is induced by KSHV and is required for infected cell survival [26]. Cell death induced by knockdown of MondaA in latently infected endothelial cells can be prevented by the direct addition of alpha-ketoglutarate to infected cells showing that induction of glutaminolysis by MondoA is necessary for infected cell survival and not a secondary effect. MondoA activation is required for the induction of the glutamine transporter, ASCT2, also known as SLC1A5. Knockdown of SLC1A5 also led to death specifically in the latently infected cells [26]. Thus, during latent infection, KSHV activates the Myc pathway and MondoA to induce glutamine uptake and glutaminolysis to produce TCA cycle intermediates.

\section{Why has KSHV evolved to induce metabolism during latency?}

The question remains as to why cancer cell-like metabolism is required for the survival of cells latently infected with KSHV. In cancer cells, the induction of glycolysis and fatty acid synthesis is thought to serve multiple roles: rapid energy production, synthesis of membrane material or other specific metabolites necessary for increased cell division, and/or increased biomass for cell growth $[8,27]$. However, KSHV does not induce rapid proliferation of endothelial cells in culture. It is possible that alterations in metabolism induced by KSHV during latency are simply a response to stress induced by viral infection. However, if this were the case, it would be expected that metabolism would change similarly during infection by many viruses. We, and others, have shown that while there are some common pathways, there are also virus specific changes to metabolism upon infection [28-30]. Alternatively, altered metabolism in latently infected cells could prepare the cell for rapid reactivation from latency. During lytic replication, CMV is known to induce and require some of the same metabolic pathways that KSHV does during latent infection [31,32]. The alterations of metabolism during latency could provide a cellular environment that is primed for reactivation, allowing rapid production of virus. We recently found that KSHV also requires glycolysis, fatty acid synthesis and glutaminolysis for lytic replication (Sanchez and Lagunoff, in preparation). However, it is seems implausible that KSHV would evolve to alter metabolism during life-long latent infection simply to prepare for reactivation. 
The changes in cellular metabolism during latency are more likely to be required for survival in changing cellular environments. Classic KS most often occurs in the lower extremities of elderly men, a known hypoxic environment [33]. The Warburg effect would allow a latently infected cell to survive and proliferate in this low oxygen environment. The induction of fatty acid synthesis could allow rapid adaptation to a cellular environment that stimulated rapid growth of latently infected cells. While KSHV induced growth and proliferation in cultured cells is not generally apparent [34], the KS tumor environment might support rapid cell division. That being said, it is hard to imagine that KSHV has evolved to cause KS, as it is not a good source of spread to new hosts. A more attractive possibility is that cells infected with KSHV require a specific metabolite to support latency. It is conceivable that to induce a few key metabolites, dramatic changes in several metabolic pathways are necessary, acting essentially as by-products. Future work will be required to distinguish the main role of altered cellular metabolism to support the survival or maintenance of KSHV latency in endothelial cells.

\section{Future considerations}

Currently there is no treatment for latent infection of herpesviruses. The approved drugs used to treat herpesviruses target only lytic replication of the virus and are unable to specifically target latently infected cells. The limited gene expression of herpesviruses during latent infection makes targeting latent virus extremely difficult. Therefore, it may be more fruitful to target specific pathologic changes to the host cell during latent infection to eliminate latently infected cells. There are a number of inhibitors of specific metabolites or metabolic enzymes currently in clinical trials for cancer treatment [13]. We have demonstrated that inhibition of specific metabolic pathways activated during KSHV latency lead to apoptosis of the latently infected cells while sparing the uninfected cells [15,25,26]. Therefore, a deeper understanding of the specific metabolic pathways required for the survival of latently infected cells could lead to novel therapeutic targets to treat diseases that occur during latent infection. For example, there are a number of cellular glutamine transporters but KSHV requires SLC1A5 for survival and, therefore, this transporter could be a therapeutic target with minimal side effects in cells that are less reliant on glutamine uptake or can utilize other glutamine transporters [26]. Targeting specific metabolites or metabolic enzymes activated by KSHV and necessary for the survival of latently infected cells will allow therapeutic targeting of latent infection with fewer side effects on the uninfected cells with normal cellular metabolism.

Future studies to identify the viral and cellular mechanisms utilized by KSHV to alter metabolism will serve not only to provide novel therapeutic targets for latency but could help future studies on how cancer cell metabolism is controlled. Cancer metabolism is established over the course of a normal cell developing into a transformed cell. However, KSHV infection of cells alters metabolism rapidly, within 24 hours, and it is therefore possible to identify the mechanisms of metabolic changes and the specific viral gene or genes responsible. The studies of viral induced metabolism could facilitate the identification of key control points for the alteration of metabolism and these are likely to be relevant for cancer cell metabolism studies. In summary, the study of how KSHV latent infection and 
other viruses alter metabolism could lead to novel viral and cancer therapeutics that target pathologic changes in metabolism.

\section{Acknowledgments}

This work was supported by a grants from the National Cancer Institute (RO1CA189986) and from the National Dental and Craniofacial Institute (PO1DE02195). The content is solely the responsibility of the authors and does not necessarily represent the official views of the National Institutes of Health.

\section{References}

1. Lagunoff M, Carroll PA. Inhibition of apoptosis by the gamma-herpesviruses. Int Rev Immunol. 2003; 22:373-399. [PubMed: 12959751]

2. Jones C. Bovine Herpes Virus 1 (BHV-1) and Herpes Simplex Virus Type 1 (HSV-1) Promote Survival of Latently Infected Sensory Neurons, in Part by Inhibiting Apoptosis. J Cell Death. 2013; 6:1-16. [PubMed: 25278776]

3. Reisman D, Sugden B. trans activation of an Epstein-Barr viral transcriptional enhancer by the Epstein-Barr viral nuclear antigen 1. Mol Cell Biol. 1986; 6:3838-3846. [PubMed: 3025615]

4. Rawlins DR, Milman G, Hayward SD, Hayward GS. Sequence-specific DNA binding of the Epstein-Barr virus nuclear antigen (EBNA-1) to clustered sites in the plasmid maintenance region. Cell. 1985; 42:859-868. [PubMed: 2996781]

5. Ballestas ME, Chatis PA, Kaye KM. Efficient persistence of extrachromosomal KSHV DNA mediated by latency-associated nuclear antigen. Science. 1999; 284:641-644. [PubMed: 10213686]

6. Wabinga HR, Nambooze S, Amulen PM, Okello C, Mbus L, Parkin DM. Trends in the incidence of cancer in Kampala, Uganda 1991-2010. Int J Cancer. 2014; 135:432-439. [PubMed: 24615279]

7. Warburg O. On respiratory impairment in cancer cells. Science. 1956; 124:269-270. [PubMed: 13351639]

8. DeBerardinis RJ, Lum JJ, Hatzivassiliou G, Thompson CB. The biology of cancer: metabolic reprogramming fuels cell growth and proliferation. Cell Metab. 2008; 7:11-20. [PubMed: 18177721]

9. Currie E, Schulze A, Zechner R, Walther TC, Farese RV Jr. Cellular fatty acid metabolism and cancer. Cell Metab. 2013; 18:153-161. [PubMed: 23791484]

10. DeBerardinis RJ, Cheng T. Q's next: the diverse functions of glutamine in metabolism, cell biology and cancer. Oncogene. 2010; 29:313-324. [PubMed: 19881548]

11. Hirschey MD, DeBerardinis RJ, Diehl AM, Drew JE, Frezza C, Green MF, Jones LW, Ko YH, Le A, Lea MA, et al. Dysregulated metabolism contributes to oncogenesis. Semin Cancer Biol. 2015; 35(Suppl):S129-150. [PubMed: 26454069]

12. Hanahan D, Weinberg RA. Hallmarks of cancer: the next generation. Cell. 2011; 144:646-674. [PubMed: 21376230]

13. Vander Heiden MG. Targeting cancer metabolism: a therapeutic window opens. Nat Rev Drug Discov. 2011; 10:671-684. [PubMed: 21878982]

14. Schulze A, Harris AL. How cancer metabolism is tuned for proliferation and vulnerable to disruption. Nature. 2012; 491:364-373. [PubMed: 23151579]

**15. Delgado T, Carroll PA, Punjabi AS, Margineantu D, Hockenbery DM, Lagunoff M. Induction of the Warburg effect by Kaposi's sarcoma herpesvirus is required for the maintenance of latently infected endothelial cells. Proc Natl Acad Sci U S A. 2010; 107:10696-10701. This was the first example of a latent virus altering metablism and a directly oncogenic virus inducing the Warburg effect. [PubMed: 20498071]

16. Carroll PA, Kenerson HL, Yeung RS, Lagunoff M. Latent Kaposi's sarcoma-associated herpesvirus infection of endothelial cells activates hypoxia-induced factors. J Virol. 2006; 80:10802-10812. [PubMed: 16956952]

17. Ma T, Patel H, Babapoor-Farrokhran S, Franklin R, Semenza GL, Sodhi A, Montaner S. KSHV induces aerobic glycolysis and angiogenesis through HIF-1-dependent upregulation of pyruvate kinase 2 in Kaposi's sarcoma. Angiogenesis. 2015; 18:477-488. [PubMed: 26092770] 
18. Xiao L, Hu ZY, Dong X, Tan Z, Li W, Tang M, Chen L, Yang L, Tao Y, Jiang Y, et al. Targeting Epstein-Barr virus oncoprotein LMP1-mediated glycolysis sensitizes nasopharyngeal carcinoma to radiation therapy. Oncogene. 2014; 33:4568-4578. [PubMed: 24662831]

19. Sommermann TG, O’Neill K, Plas DR, Cahir-McFarland E. IKKbeta and NF-kappaB transcription govern lymphoma cell survival through AKT-induced plasma membrane trafficking of GLUT1. Cancer Res. 2011; 71:7291-7300. [PubMed: 21987722]

*20. Bhatt AP, Jacobs SR, Freemerman AJ, Makowski L, Rathmell JC, Dittmer DP, Damania B. Dysregulation of fatty acid synthesis and glycolysis in non-Hodgkin lymphoma. Proc Natl Acad Sci U S A. 2012; 109:11818-11823. Showed that KSHV Infected primary effusion lymphomas maintina high levels of glycolysis and fatty acid synthesis. [PubMed: 22752304]

21. McFadden K, Hafez AY, Kishton R, Messinger JE, Nikitin PA, Rathmell JC, Luftig MA. Metabolic stress is a barrier to Epstein-Barr virus-mediated B-cell immortalization. Proc Natl Acad Sci U S A. 2016; 113:E782-790. [PubMed: 26802124]

22. Darekar S, Georgiou K, Yurchenko M, Yenamandra SP, Chachami G, Simos G, Klein G, Kashuba E. Epstein-Barr virus immortalization of human B-cells leads to stabilization of hypoxia-induced factor 1 alpha, congruent with the Warburg effect. PLoS One. 2012; 7:e42072. [PubMed: 22848707]

*23. Yogev O, Lagos D, Enver T, Boshoff C. Kaposi’s sarcoma herpesvirus microRNAs induce metabolic transformation of infected cells. PLoS Pathog. 2014; 10:e1004400. Found that a KSHV miRNA cluster was sufficient to induce glycolysis. [PubMed: 25255370]

24. Lo AK, Dawson CW, Young LS, Ko CW, Hau PM, Lo KW. Activation of the FGFR1 signalling pathway by the Epstein-Barr virus-encoded LMP1 promotes aerobic glycolysis and transformation of human nasopharyngeal epithelial cells. J Pathol. 2015; 237:238-248. [PubMed: 26096068]

**25. Delgado T, Sanchez EL, Camarda R, Lagunoff M. Global metabolic profiling of infection by an oncogenic virus: KSHV induces and requires lipogenesis for survival of latent infection. PLoS Pathog. 2012; 8:e1002866. First global metabolomic screen of cells during latent infection with a herpesvirus and showed that latently infected cells require fatty acid synthesis. [PubMed: 22916018]

**26. Sanchez EL, Carroll PA, Thalhofer AB, Lagunoff M. Latent KSHV Infected Endothelial Cells Are Glutamine Addicted and Require Glutaminolysis for Survival. PLoS Pathog. 2015; 11:e1005052. First example that cells latently infecgted with KSHV require glutamine for anaplerosis and showed the importance of MondoA for induction of a glutamine transporter that is required. [PubMed: 26197457]

27. Cantor JR, Sabatini DM. Cancer cell metabolism: one hallmark, many faces. Cancer Discov. 2012; 2:881-898. [PubMed: 23009760]

28. Vastag L, Koyuncu E, Grady SL, Shenk TE, Rabinowitz JD. Divergent effects of human cytomegalovirus and herpes simplex virus-1 on cellular metabolism. PLoS Pathog. 2011; 7:e1002124. [PubMed: 21779165]

*29. Sanchez EL, Lagunoff M. Viral activation of cellular metabolism. Virology. 2015; 479-480:609618. Overall review of viruses and metabolism focusing on the many viruses that induce glycolysis, fatty acid synthesis and glutaminolysis.

30. Fontaine KA, Camarda R, Lagunoff M. Vaccinia virus requires glutamine but not glucose for efficient replication. J Virol. 2014; 88:4366-4374. [PubMed: 24501408]

31. Munger J, Bajad SU, Coller HA, Shenk T, Rabinowitz JD. Dynamics of the cellular metabolome during human cytomegalovirus infection. PLoS Pathog. 2006; 2:e132. [PubMed: 17173481]

32. Munger J, Bennett BD, Parikh A, Feng XJ, McArdle J, Rabitz HA, Shenk T, Rabinowitz JD. Systems-level metabolic flux profiling identifies fatty acid synthesis as a target for antiviral therapy. Nat Biotechnol. 2008; 26:1179-1186. [PubMed: 18820684]

33. Davis DA, Rinderknecht AS, Zoeteweij JP, Aoki Y, Read-Connole EL, Tosato G, Blauvelt A, Yarchoan R. Hypoxia induces lytic replication of Kaposi sarcoma-associated herpesvirus. Blood. 2001; 97:3244-3250. [PubMed: 11342455]

34. Lagunoff M, Bechtel J, Venetsanakos E, Roy AM, Abbey N, Herndier B, McMahon M, Ganem D. De novo infection and serial transmission of Kaposi's sarcoma-associated herpesvirus in cultured endothelial cells. J Virol. 2002; 76:2440-2448. [PubMed: 11836422] 


\section{Highlights}

- $\quad$ KSHV latent infection of endothelial cells activates glycolysis, fatty acid synthesis and glutaminolysis

- Inhibitors of each of these metabolic pathways leads to apoptotic cell death of latently infected endothelial cells

- $\quad$ Metabolic inhibitors may be useful to treat latent infection of KSHV

- $\quad$ KSHV induction of cancer cell-like metabolism could provide novel keys to understanding the control of cancer cell metabolism 


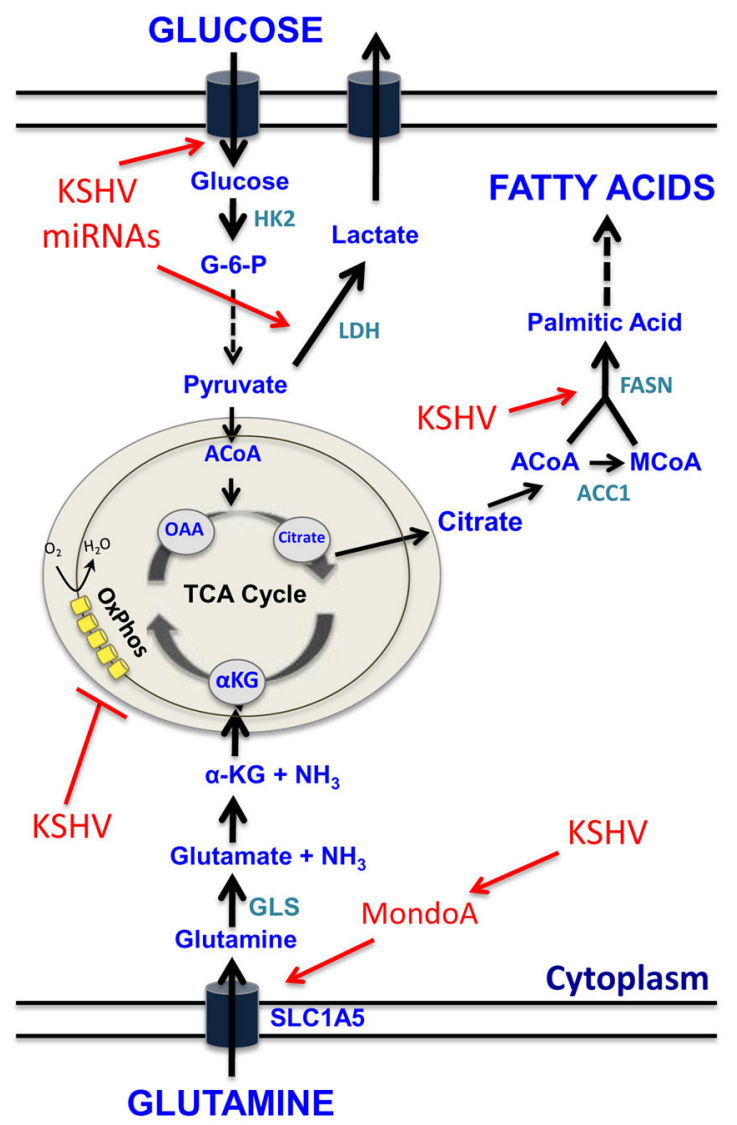

Figure 1. Depiction of the three major metabolic pathways, glycolysis, glutaminolysis and fatty acid synthesis, that are altered in many cancer cells and by latent KSHV infection

A subset of key metabolites in the three major metabolic pathways and the TCA cycle are shown in blue. During latency, KSHV activates all three major pathways at points indicated by red arrows and inhibits oxidative phosphorylation. Chemical inhibition of the metabolic enzymes shown in light blue has been shown to lead to cell death in latently infected endothelial cells. 